\title{
Generalized Order Statistics from Generalized Exponential Distributions in Explicit Forms
}

\author{
Hazem I. El Shekh Ahmed \\ Al Quds Open University, Gaza, Palestine \\ Email: hshaikhahmad@qou.edu
}

Received January 6, 2013; revised February 5, 2013; accepted February 20, 2013

Copyright (C) 2013 Hazem I. El Shekh Ahmed. This is an open access article distributed under the Creative Commons Attribution License, which permits unrestricted use, distribution, and reproduction in any medium, provided the original work is properly cited.

\begin{abstract}
The generalized order statistics which introduced by [1] are studied in the present paper. The Gompertz distribution is widely used to describe the distribution of adult deaths, and some related models used in the economic applications [2]. Previous works concentrated on formulating approximate relationships to characterize it [3-5]. The main aim of this paper is to obtain the distribution of single, two, and all generalized order statistics from Gompertz distribution with some special cases. In addition the conditional distribution of two generalized order statistics from the same distribution is obtained. The Gompertz distribution has a continuous probability density function with location parameter a and shape parameter $b, f(x)=a \mathrm{e}^{b x-\frac{a}{b}\left(e^{b x-1}\right)}$, where $x$ restricted by the interval $[0, \infty)$. The $n$th moment generated function of the Gompertz distributed random variable $X$ is given on the form: $E\left(X^{n}\right)=\frac{n !}{b^{n}} \mathrm{e}^{\frac{a}{b}} E_{1}^{n-1}\left(\frac{a}{b}\right)$, where, $E_{s}^{n}(z)=\frac{1}{n !} \int_{1}^{\infty}(\ln x)^{n} x^{-s} \mathrm{e}^{-z x} \mathrm{~d} z$ is the generalized integro-exponential function [6]. In this paper we shall obtain joint distribution, distribution of product of two generalized order statistics from the Gompertz distribution, and then derive
\end{abstract} some useful formulas of these distributions as special cases.

Keywords: Gompertz Distribution; kth Record Model Values; Generalized Order Statistics

\section{Introduction}

Order statistics appears in many statistical applications and is widely used in statistical modeling and inference. Such models describe random variables arranged in ascending order of magnitude. In a wide subclass of generalized order statistics, representations of marginal, joint and probability density distribution functions are developed. The results are applied to obtain these representations for several expressions for the joint of generalized order statistics from Exponential Pareto distribution [7].

The Gompertz distribution plays an important role in modeling survival times, human mortality and actuarial tables. According to the literature, the Gompertz distribution was formulated by Gompertz (1825) to fit mortality tables. On the other hand, generalized order statistics (GOS) have been of interest in the past ten years because they are more flexible in reliability theory, statistical modeling and inference, the generalized order statistics have been introduced as a unified distribution theore- tical set-up which contains a variety of models of ordered random variables with different interpretations. The subject of order statistics has been further generalized and the concept of generalized order statistics (GOS) is introduced and studied by Kamps in a series of papers and books [1,8-10].

The ordered random variables such as order statistics play an important role in many branches of statistics and applied probability. Kamps in [9] introduced the concept of GOS and showed that order statistics, record values, and some other ordered random variables can be considered as special cases of generalized order statistics. M. Ragab, in [11] established several recurrence relations satisfied by the single and the product moments for order statistics from the Generalized Exponential Distribution (GED). The relationships can be written in terms of polygamma and hypergeometric functions and used in a simple recursive manner in order to compute the single and the product moments of all order statistics for all 
sample sizes.

The Generalized Exponential Distribution (GED) with two non-negative parameters $\theta$ and $\lambda$ is considered to be one of those distributions which have real attention from researchers. It has been studied and introduced by [12].

In the present paper we shall obtain joint distribution and distribution of product of two generalized order statistics from the Gompertz distribution.

\section{Generalized Order Statistics (GOS)}

Generalized order statistics (GOS) have been of interest in the past ten years because they are more flexible in reliability theory, statistical modeling and inference [12], Uniform generalized order statistics is defined via some joint density function on a cone of Rn. (GOS) based on an arbitrary distribution function $\mathrm{F}$ is defined by means of the inverse function of $F$, as in the following:

Definition (1) Let $F(x)$ denotes an absolutely continuous distribution function with density function $f(x)$, then the sequence of random variables

$X_{1: n, m, k}, X_{2: n, m, k}, \cdots, X_{n: n, m, k}$ is called " $n$ " Generalized Order Statistics (GOS), where ( $k \geq 1, \mathrm{~m}$ is a real number) [3].

Definition (2) The random variables

$X(1, n, \tilde{m}, k), \cdots, X(n, n, \tilde{m}, k)$ are called GOS based on the cumulative distribution function (cdf), $F(x)$, if their joint probability density function (pdf) is given by

$$
\begin{aligned}
& f_{1, \cdots, n}\left(x_{1}, \cdots, x_{n}\right) \\
& =\left\{\begin{array}{l}
k\left(\prod_{j=1}^{n-1} \gamma_{j}\right)\left[\prod_{i=1}^{n-1}\left(1-F\left(x_{i}\right)\right)^{m} f\left(x_{i}\right)\right] \\
\cdot\left(1-F\left(x_{n}\right)\right)^{k-1} f\left(x_{n}\right), \\
\text { for } F^{-1}(0)<x_{1}<\cdots<x_{n}<F^{-1}(1) \\
0, \quad \text { otherwise }
\end{array}\right.
\end{aligned}
$$

with parameters $n \in N, n \geq 2, k>0$, $\tilde{m}=\left(m_{1}, m_{2}, \cdots, m_{n-1}\right), M_{r}=\sum_{j=r}^{n-1} m_{j}$, such that $\gamma_{r}=k+n-r+M_{r}>0$ for all $r \in\{1, \cdots, n-1\}$, let $c_{r-1}=\prod_{j=1}^{r} \gamma_{j}, r=1,2, \cdots, n-1$ and $\gamma_{n}=k$.

The cumulative distribution function (cdf) of such generalized order statistics random variable $X(r, n, m, k)$ as,

$$
F_{1, \cdots, n}(X)=1-c_{r-1} \sum_{j=1}^{r} \frac{a_{j}(r)}{\gamma_{j}}(1-F(X))^{\gamma_{j}}
$$

where $a_{j}(r)=\prod_{i=1, i \neq j}^{r}\left(\frac{1}{\gamma_{i}-\gamma_{j}}\right)$ or $1 \leq j \leq r \leq n$
Now, if $m=0$ and $k=1$ it gives the joint pdf of " $n$ " ordinary order statistics $X_{1, n}, X_{2, n}, \cdots, X_{n, n}$, moreover if $m=-1, k=1$, it gives the joint pdf of the first " $n$ " upper records of the independent identically distributed random variables.

\section{The Gompertz Distribution}

A random variable $\mathrm{X}$ is said to have Gompertz distribution with location parameter a and shape parameter $b$, has a continuous probability density function (pdf) and formulated as,

$$
f(x)=a \mathrm{e}^{b x-\frac{a}{b}\left(\mathrm{e}^{b x-1}\right)}
$$

with a supported domain on $(-\infty, \infty)$.

The distribution function (cdf) is:

$$
F(x)=1-\mathrm{e}^{-\frac{a}{b}\left(\mathrm{e}^{b x-1}\right)}
$$

\section{Joint Distribution of All Generalized Order Statistics}

\subsection{Joint Distribution of All Generalized Order Statistics for Generalized Gompertz Distribution (GOSGD)}

The generalized order statistics were defined by [14] as follows:

Definition (3) Let $n \in N, k>0, \tilde{m}=\left(m_{1}, \cdots, m_{n-1}\right) \in R^{n-1}$ be parameters such that $\gamma_{r}=k+n-r+\sum_{g=r}^{n-1} m_{j}>0$ for all $r \in\{1, \cdots, n\}$. If the random variables $U(r, n, \tilde{m}, k), r=1, \cdots, n$, possess a joint density function of the form:

$$
\begin{aligned}
& f^{U(1, n, \tilde{m}, k), \cdots, U(n, n, \tilde{m}, k)}\left(u_{1}, \cdots, u_{n}\right) \\
& =k\left(\prod_{j=1}^{n-1} \gamma_{j}\right)\left[\prod_{i=1}^{n-1}\left(1-u_{i}\right)^{m_{i}}\right]\left(1-u_{n}\right)^{k-1},
\end{aligned}
$$

on the cone $0 \leq u_{1} \leq \cdots \leq u_{n}<1$ of $R^{n}$, then they are called Uniform generalized order statistics. The random variables $X(1, n, \tilde{m}, k)=F^{-1}(U(r, n, \tilde{m}, k))$ are called generalized order statistics (based on $F$ ), which have a joint density function of the form

$$
\begin{aligned}
& f^{X(1, n, \tilde{m}, k), \cdots, X(n, n, \tilde{m}, k)}\left(x_{1}, \cdots, x_{n}\right) \\
& =k\left(\prod_{j=1}^{n-1} \gamma_{j}\right)\left[\prod_{i=1}^{n-1}\left(1-F\left(x_{i}\right) f\left(x_{i}\right)\right)^{m_{i}}\right] \\
& \cdot\left(1-F\left(x_{n}\right)\right)^{k-1} f\left(x_{n}\right)
\end{aligned}
$$

where, $F^{-1}(0)<X_{1} \leq X_{2} \leq \cdots \leq X_{n}<F^{-1}(1)$.

The marginal density function of $r^{\text {th }}$ GOS

$X(r, n, \tilde{m}, k), r=1, \cdots, n$ based on $F$ is formulated by [13] as, 


$$
f_{X(r, n, m, k)}(x)=\phi_{r: n}(F(x)) f(x)
$$

where

$$
\begin{aligned}
& \phi_{r: n}(x)=\frac{C_{r-1}}{(r-1) !}(1-x)^{\gamma_{r}-1} g_{m}^{r-1}(x), \\
& x \in(0,1), \\
& g_{m}(x)=\left\{\begin{array}{lr}
\frac{1}{m+1}\left[1-(1-x)^{m+1}\right], & \text { if } m \neq-1 \\
-\ln (1-x), & \text { if } m=-1
\end{array}\right. \\
& x \in[0,1)
\end{aligned}
$$$$
\text { and } c_{r-1}=\prod_{i=1}^{r} \gamma_{i} \text {. }
$$

The corresponding marginal distribution of $r^{\text {th }}$ GOS based on $F$ is

$$
F_{X(r, n, m, k)}(x)=\phi_{r: n}(F(x)),
$$

where

$$
\begin{aligned}
& \phi_{r: n}(x)=1-\sum_{i=0}^{r-1} \frac{c_{i-1}}{i !}(1-u)^{\gamma_{r}+1}\left[g_{m}(u)\right]^{i}, \\
& u \in(0,1),
\end{aligned}
$$

We discuss some special cases in Corollaries 2.2 and 2.3 .

Corollary 4.1 (The joint pdf of all ordinary order statistics for Gompertz Distribution)

In Equation (5), let $k=1$ and $m=0$, then the joint pdf of all ordinary order statistics

$X(1, n, 0,1), \cdots, X(n, n, 0,1)$ for Gompertz distribution is

$$
\begin{aligned}
& f\left(x_{1}, \cdots, x_{n}\right) \\
& =\left[\prod_{j=1}^{n-1} \gamma_{j}\right]\left[\prod_{i=1}^{n-1} a \mathrm{e}^{\left.b x_{i}-\frac{a}{b} \mathrm{e}^{b x_{i}}-1\right)}\right] a \mathrm{e}^{b x_{n}-k \frac{a}{b}\left(\mathrm{e}^{b x_{n}}-1\right)}
\end{aligned}
$$

Again in Equation (1), let $k=1$ and $m=0$, then one can directly see that

$\prod_{j=1}^{n-1} \gamma_{j}=\prod_{j=1}^{n-1}(1+n-j)=n \times(n-1) \cdots 3 \times 2 \times 1=n$ !. Therefore the joint pdf of all ordinary order statistics for Gompertz Distribution

$$
f\left(x_{1}, \cdots, x_{n}\right)=n !\left[\prod_{i=1}^{n-1} a \mathrm{e}^{b x_{i}-\frac{a}{b}\left(\mathrm{e}^{b x_{i}}-1\right)}\right] a \mathrm{e}^{b x_{n}-k \frac{a}{b}\left(\mathrm{e}^{b x_{n}}-1\right)}
$$
2.6 .

We discuss some special cases in Corollaries 2.5 and

\subsection{Joint Distribution of Two Generalized Order Statistics}

The joint pdf of $i^{\text {th }}$ and $j^{\text {th }}$ generalized order statistics, $X(i, n, \tilde{m}, k)$ and $X(j, n, \tilde{m}, k)$, is given by

$$
\begin{aligned}
& f_{i, j, n, \tilde{m}, k}\left(x_{i}, x_{j}\right) \\
& =\frac{c_{j}}{(i-1) !(j-i-1) !}\left[1-F\left(x_{i}\right)\right]^{m} \\
& \cdot\left[1-F\left(x_{j}\right)\right]^{\gamma_{i}-1}\left[g_{m}\left(F\left(x_{i}\right)\right)\right]^{i-1} D
\end{aligned}
$$

where

$$
D=\left[g_{m}\left(F\left(x_{j}\right)\right)-g_{m}\left(F\left(x_{i}\right)\right)\right]^{j-i-1} f\left(x_{i}\right) f\left(x_{j}\right),
$$

for $0<x_{i}<x_{j}<\infty, 1 \leq i<j \leq n$, where,

$$
c_{r}=\prod_{j=1}^{r} \gamma_{j}, \gamma_{j}=k+(n-j)(m+1) .
$$

Since $\lim _{m \rightarrow-1}\left(\frac{1-(1-x)^{m+1}}{m+1}\right)=-\ln (1-x)$, we shall write $g_{m}(x)=\frac{1-(1-x)^{m+1}}{m+1}$ for all $x \in(0,1)$ and for all $m$ with $g_{-1}(x)=\lim _{m \rightarrow-1} g_{m}(x)$, see [11]

\subsection{Joint Distribution of Two Generalized Order Statistics for Generalized Gompertz Distribution}

In the next theorem 4.1, we will derive the joint pdf of $X(i, n, \tilde{m}, k)$ and $X(j, n, \tilde{m}, k)$ for GOSGD.

Theorem 4.1 The joint probability density function of $X(i, n, \tilde{m}, k)$ and $X(j, n, \tilde{m}, k)$ for Gompertz distribution can be formulated by,

$$
\begin{aligned}
& f_{i, j, n, \tilde{m}, k}\left(x_{i}, x_{j}\right) \\
& =\frac{c_{j}}{(i-1)(j-i-1) !}\left[\mathrm{e}^{-\frac{a}{b}\left(b x_{i}-1\right)}\right]^{m}\left[\mathrm{e}^{-\frac{a}{b}\left(b x_{j}-1\right)}\right]^{\gamma_{j-1}} \\
& \cdot\left[\frac{1-\left(\mathrm{e}^{-\frac{a}{b}\left(b x_{i}-1\right)}\right)^{m+1}}{m+1}\right]^{i-1} D
\end{aligned}
$$

where

$$
\begin{aligned}
& D=\left[\left(\mathrm{e}^{-\frac{a}{b}\left(\mathrm{e}^{\left(x_{i}-1\right.}\right)}\right)^{m+1}-\left(\mathrm{e}^{-\frac{a}{b}\left(\mathrm{e}^{b x_{j}-1}\right)}\right)^{m+1}\right]^{j-i-1} \\
& \cdot a^{2} \mathrm{e}^{b\left(x_{i}+x_{j}\right)-\frac{a}{b}\left[\mathrm{e}^{\left[\left(b\left(x_{i}-x_{j}\right)-2\right)\right.}\right]}
\end{aligned}
$$

for $0<x_{i}<x_{j}<\infty, 1 \leq i<j \leq n$, where,

$$
c_{r}=\prod_{j=1}^{r} \gamma_{j}, \gamma_{j}=1+(n-j) \text {. }
$$

Proof:

The proof can be established by Equations (3), (4), and (10) to obtain the formula (11) 


\section{Special cases:}

1) The joint density function of two ordinary Order Statistics for Gompertz Distribution

Let $k=1$ and $m=0$, then Equation (11) reduces joint density of two ordinary order statistics $X(i, n, 0,1), X(j, n, 0,1)$ in Gompertz distribution as,

$$
\begin{aligned}
& f_{i, j, n, 0,1}\left(x_{i}, x_{j}\right) \\
& =\frac{c_{j}}{(i-1)(j-i-1) !}\left[\mathrm{e}^{-\frac{a}{b}\left(b x_{j}-1\right)}\right]^{\gamma_{j-1}}\left[1-\left(\mathrm{e}^{-\frac{a}{b}\left(b x_{i}-1\right)}\right)\right]^{i-1} D
\end{aligned}
$$

where

$$
\begin{aligned}
& D=\left[\left(\mathrm{e}^{-\frac{a}{b}\left(\mathrm{e}^{b x_{i}-1}\right)}\right)-\left(\mathrm{e}^{-\frac{a}{b}\left(\mathrm{e}^{\left(x_{j}-1\right.}\right)}\right)\right]^{j-i-1} \\
& \cdot a^{2} \mathrm{e}^{b\left(x_{i}+x_{j}\right)-\frac{a}{b}\left[\left(\mathrm{e}^{b\left(x_{i}-x_{j}\right)-2}\right)\right]}
\end{aligned}
$$

2) The $k$ th record model values in (GOSGD)

For any $k \in N$ and $m=-1$, then Equation (6) directly reduces the $k$ th record model in GOSGD with $g_{-1}(x)=-\ln (1-x)$.

\section{Distribution of Single Generalized Order Statistics}

In the following, the concept single generalized order statistics is presented and then going to illustrate the equivalent formulas for the Gompertz distribution.

Definition (4) Further integrating out

$x_{1}, \cdots, x_{r-1}, x_{r+1}, \cdots, x_{n}$ from Definition (2), we get the pdf $f_{r, n, \tilde{m}, k}$ of $X(r, n, m, k)$ as

$$
\begin{aligned}
& f_{r, n, \tilde{m}, k}(x) \\
& =\frac{c_{r}}{(r-1) !}\left[1-F\left(x_{r}\right)\right]^{\gamma_{r}-1} g_{m}^{r-1}\left(F\left(x_{r}\right)\right) f\left(x_{r}\right)
\end{aligned}
$$

where, $c_{r}=\prod_{j=1}^{r} \gamma_{j}, \gamma_{r}=k+(n-r)(m+1)$ and

$$
g_{m}(x)=\frac{1}{m+1}\left[1-(1-x)^{m+1}\right]
$$

for all $x \in(0,1)$ for all $m$, with $g_{-1}(x)=\lim _{x \rightarrow-1} g_{m}(x)$, see [14].

Lemma 5.1 The pdf of the minimum generalized order statistic is

$$
\begin{aligned}
& f_{1, n, \tilde{m}, k}(x) \\
& =[k+(n-1)(m+1)]\left[1-F\left(x_{1}\right)\right]^{k+(n-1)(m+1)-1} f\left(x_{1}\right)
\end{aligned}
$$

\section{Proof:}

Using Equation (13), let $r=1$, then $c_{1}=\gamma_{1}=k+(n-1)(m+1)$, we get Equation (14) and that completes the proof.

Lemma 5.2 The pdf of the maximum generalized order statistic is

$$
\begin{aligned}
& f_{n, n, \tilde{m}, k}(x) \\
& =\frac{[k+(n-1)(m+1)][k+(n-2)(m+1)] \cdots[k]}{(n-1) !} \\
& \cdot\left[1-F\left(x_{n}\right)\right]^{k-1} \\
& \times\left(\frac{1}{m+1}\left[1-\left(1-F\left(x_{n}\right)\right)^{m+1}\right]\right)^{n-1} f\left(x_{n}\right)
\end{aligned}
$$

Proof:

Using Definition (4), let $r=n$, then

$$
\begin{aligned}
& g_{m}\left(F\left(x_{n}\right)\right)=\frac{1}{m+1}\left[1-\left(1-F\left(x_{n}\right)\right)^{m+1}\right], \gamma_{n}=k \\
& c_{n}=\prod_{j=1}^{n} k+(n-j)(m+1) \\
& =[k+(n-1)(m+1)][k+(n-2)(m+1)] \ldots[k],
\end{aligned}
$$

we get Equation (15) and that completes the proof.

\section{Distribution of Single Generalized Order Statistics for Gompertz Distribution}

Lemma 5.3 Using the pdf and cdf given in Equations (2) and (3) in Equation (13), and collecting terms we get

$$
\begin{aligned}
f_{r, n, \tilde{m}, k}(x) \\
=\frac{c_{r}}{(r-1) !}\left[\mathrm{e}^{-\frac{a}{b}\left(\mathrm{e}^{b x r}-1\right)}\right]^{\gamma_{r}-1}\left(\frac{1}{m+1}\left[1-\left[\mathrm{e}^{-\frac{a}{b}\left(\mathrm{e}^{b x}-1\right)}\right]^{m+1}\right]\right)^{r-1} \\
\times a \mathrm{e}^{b x r-\frac{a}{b}\left(\mathrm{e}^{b x r}-1\right)}
\end{aligned}
$$

Corollary 5.1 The pdf of the minimum generalized order statistic for Gompertz Distribution

In Equation (16), let $r=1$, $\left[c_{1}=\gamma_{1}=k+(n-1)(m+1)\right]$, and collecting terms then the pdf of the minimum generalized order statistic for Gompertz Distribution is

$$
\begin{aligned}
& f_{1, n, \tilde{m}, k}(x) \\
& =[k+(n-1)(m+1)] \\
& \quad \times\left[\mathrm{e}^{-\frac{a}{b}\left(\mathrm{e}^{b x_{i}}-1\right)}\right]^{k+(n-1)(m+1)-1} a \mathrm{e}^{b x_{i}-\frac{a}{b}\left(\mathrm{e}^{b x_{i}}-1\right)}
\end{aligned}
$$

Corollary 5.2 The pdf of the minimum ordinary statistics for Gompertz Distribution

In Equation (17), let $k=1$ and $m=0$ then,

$$
f_{1, n, 0,1}(x)=[1+(n-1)]\left[\mathrm{e}^{-\frac{a}{b}\left(\mathrm{e}^{b x_{i}}-1\right)}\right]^{n-1} a \mathrm{e}^{b x_{i}-\frac{a}{b}\left(\mathrm{e}^{b x_{i}}-1\right)}
$$

Lemma 5.3 Using the pdf and cdf given in Equations (2) and (3) in Equation (15), and collecting terms we get 


$$
\begin{aligned}
f_{n, n, \tilde{m}, k}(x) & =\frac{[k+(n-1)(m+1)][k+(n-2)(m+1)] \cdots[k]}{(n-1) !}\left(\mathrm{e}^{-\frac{a}{b}\left(\mathrm{e}^{b x_{n}}-1\right)}\right)^{k-1} \\
& \times\left(\frac{1}{m+1}\left[1-\left(\mathrm{e}^{-\frac{a}{b}\left(\mathrm{e}^{b x_{n}}-1\right)}\right)^{m+1}\right]\right)^{n-1} a \mathrm{e}^{b x_{n}-\frac{a}{b}\left(\mathrm{e}^{x_{n}}-1\right)}
\end{aligned}
$$

\section{Corollary 5.4 The pdf of the maximum generalized order statistic for Gompertz Distribution}

In Equation (18), let $k=1$ and $m=0$, then the pdf of the maximum ordinary order statistic for Gompertz Distribution

$$
f_{n, n, 0,1}(x)=n\left(1-\left(\mathrm{e}^{-\frac{a}{b}\left(\mathrm{e}^{b x_{n}}-1\right)}\right)\right)^{n-1} a \mathrm{e}^{b x_{n}-\frac{a}{b}\left(\mathrm{e}^{b x_{n}}-1\right)}
$$

where,

$$
\begin{aligned}
& \frac{[k+(n-1)(m+1)][k+(n-2)(m+1)] \cdots[k]}{(n-1) !} \\
& =\frac{n \times(n-1) \times \cdots \times 3 \times 2 \times 1}{(n-1) !}=\frac{n !}{(n-1) !}=n
\end{aligned}
$$

\section{Conditional Distribution of Generalized Order Statistics}

In this section some previous literature of the conditional distribution of generalized order statistics is presented and then derived these results for Gompertz distribution.

Theorem 5.1 (See [15])

Let $X_{1}, \cdots, X_{n}$ be a random sample from a continuous population with cdf $F(x)$ and pdf $f(x)$. Let $X(1, n, \tilde{m}, k), X(2, n, \tilde{m}, k), \cdots, X(n, n, \tilde{m}, k)$ denote the generalized order statistics obtained from this sample. Then the conditional distribution of

$X(s, n, \tilde{m}, k) \mid X(r, n, \tilde{m}, k)=x$ for $r<s$ is

$$
\begin{aligned}
& h(X(s, n, \tilde{m}, k) \mid X(r, n, \tilde{m}, k)) \\
& =\frac{c_{s}}{c_{r}(s-r-1) !} \times \frac{[1-F(x)]^{m}[1-F(y)]^{\gamma_{s}-1}\left[g_{m}(F(y))-g_{m}(F(x))\right]^{s-r-1} f(y)}{[1-F(x)]^{\gamma_{r}-1}}
\end{aligned}
$$

$F^{-1}(0)<x \leq y<F^{-1}(1)$

Notes $c_{r}=\prod_{j=1}^{r} \gamma_{j}, \gamma_{j}=k+(n-j)(m+1)$ and $g_{m}(x)=\frac{1}{m+1}\left[1-(1-x)^{m+1}\right]$ for all $x \in(0,1)$ and for all $m$ with $g_{-1}(x)=\lim _{m \rightarrow-1} g_{m}(x)$.

Corollary 5.5 (Conditional distribution of an ordinary order statistics)

Using Theorem (5.1), if $k=1$ and $m=0$, then

$$
\begin{aligned}
& h(X(s, n, 0,1) \mid X(r, n, 0,1))=\frac{[1-F(x)]^{0}[1-F(y)]^{\gamma_{s}-1}\left[g_{0}(F(y))-g_{0}(F(x))\right]^{s-r-1} f(y) c_{s}}{[1-F(x)]^{\gamma_{r}-1} c_{r}(s-r-1) !}, \\
& x(r, n, 0,1) \leq y(s, n, 0,1)<\infty \\
& \gamma_{r}=1+(n-r), \gamma_{s}=1+(n-s), \\
& \gamma_{r+1}=1+(n-r-1)=n-r \\
& g_{0}(F(y))=F(y) \\
& g_{0}(F(x))=F(x) \\
& c_{s}=n(n-1)(n-2) \cdots(1+n-s)=\frac{n !}{(n-s) !} \text {, and } \\
& c_{r}=n(n-1)(n-2) \cdots(1+n-r)=\frac{n !}{(n-r) !} . \text { Then } \\
& \begin{array}{l}
h(X(s, n, 0,1) \mid X(r, n, 0,1)) \\
=\frac{(n-r) ![1-F(y)]^{n-s}[(F(y))-(F(x))]^{s-r-1} f(y)}{(n-s) !(s-r-1) ![1-F(x)]^{n-r}},
\end{array}
\end{aligned}
$$

\section{Conditional Distribution of Generalized Order Statistics for Gompertz Distribution}




$$
\begin{aligned}
& h(X(s, n, \tilde{m}, k) \mid X(r, n, \tilde{m}, k)) \\
& =\frac{c_{s}}{c_{r}(s-r-1) !} \times \frac{\left[\mathrm{e}^{-\frac{a}{b}\left(\mathrm{e}^{b x}-1\right)}\right]^{m}\left[\mathrm{e}^{-\frac{a}{b}\left(\mathrm{e}^{b y}-1\right)}\right]^{\gamma_{s}-1}\left[\frac { 1 } { m + 1 } \left[\left(\mathrm{e}^{-\frac{a}{b}\left(\mathrm{e}^{b y}-1\right)}\right)^{m+1}-\left(\mathrm{e}^{\left.\left.\left.-\frac{a}{b}\left(\mathrm{e}^{b x}-1\right)\right)^{m+1}\right]\right]^{s-r-1}} a \mathrm{e}^{b y-\frac{a}{b}\left(\mathrm{e}^{b y}-1\right)}\right.\right.\right.}{\left[\mathrm{e}^{-\frac{a}{b}\left(\mathrm{e}^{b x}-1\right)}\right]^{\gamma_{r}-1}}
\end{aligned}
$$

\section{Corollary 5.6}

In Equation (22), let $k=1$ and $m=0$, and collecting terms then the conditional pdf of two ordinary order statistics, $X(s, n, 0,1) \mid X(r, n, 0,1)=x$, for Gompertz distribution is

$$
\begin{aligned}
& h(X(s, n, 0,1) \mid X(r, n, 0,1)) \\
& =\frac{(n-r) !}{(n-s) !(s-r-1) !} \\
& \frac{\left[\mathrm{e}^{-\frac{a}{b}\left(\mathrm{e}^{b y}-1\right)}\right]^{\gamma_{s}-1}\left[\mathrm{e}^{-\frac{a}{b}\left(\mathrm{e}^{b y}-1\right)}-\mathrm{e}^{-\frac{a}{b}\left(\mathrm{e}^{b x}-1\right)}\right]^{s-r-1} a \mathrm{e}^{b y-\frac{a}{b}\left(\mathrm{e}^{b y}-1\right)}}{\left[\mathrm{e}^{-\frac{a}{b}\left(\mathrm{e}^{b x}-1\right)}\right]^{\gamma_{r}-1}}
\end{aligned}
$$

where,

$$
\begin{aligned}
& \frac{c_{s}}{c_{r}(s-r-1) !}=\frac{(n-r) !}{(n-s) !(s-r-1) !}, \\
& -\gamma_{r}+1=r-n, \gamma_{s}=n-s+1
\end{aligned}
$$

which is the well known conditional distribution of two ordinary order statistics $X(s, n, 0,1) \mid X(r, n, 0,1)$ for LGD.

\section{Conclusion and Future Research}

In this paper, we have derived the joint pdfs of generalized order statistics for Generalized and Linear Exponential distributions in explicit forms. In addition, the pdf of the conditional distribution of generalized order statistics from those distributions is given. Furthermore, some special cases have been discussed.

Many opportunities of future research are available. The plan for the future research on generalized order Statistics from Generalized and Linear Exponential distributions can be split into two main areas. Estimation and hypothesis testing of Generalized Exponential parameters based on generalized order statistics.

\section{Acknowledgements}

The author would like to thank the lecture Shaikh Ahmed $\mathrm{R}$. for her efforts in this research and to Mr. Fayyad M. for his technical aid.

\section{REFERENCES}

[1] U. Kamps, "Characterizations of the Exponential Distribution by Weighted Sums of Iid Random Variables," Statistical Papers, Vol. 31, No. 1, 1990, pp. 233-237. doi:10.1007/BF02924695

[2] P. Jodrá, "A Closed-Form Expression for the Quantile Function of the Gompertz-Makeham Distribution," $M a-$ thematics and Computers in Simulation, Vol. 79, No. 10, 2009, pp. 3069-3075. doi:10.1016/j.matcom.2009.02.002

[3] D. Kunimura, "The Gompertz Distribution-Estimation of Parameters," Actuarial Research Clearing House, Vol. 2, 1998, pp. 65-76.

[4] F. M. Bass, "A New Product Growth Model for Consumer Durables," Management Science, Vol. 15, No. 5, 1969, pp. 215-227. doi:10.1287/mnsc.15.5.215

[5] J. Pollard and E. Valkovics, "The Gompertz Distribution and Its Applications," Genus, Vol. 48, No. 34, 1992, pp. 15-29.

[6] A. Lenart, "The Gompertz Distribution and Maximum Likelihood Estimation of Its Parameter-A Revision," Max Planck Institute for Demographic Research, Rostock, 2012.

[7] R. U. Khan and D. Kumar, "On Moments of Lower Genralized Order Statistics from Exponintial Pareto Distribution and Its Characterization," Applied Mathematical Sciences, Vol. 4, No. 55, 2010, pp. 2711-2722.

[8] U. Kamps and U. Gather, "Characteristic Properties of Generalized Order Statistics from Exponential Distributions," Applicationes Mathematicae, Vol. 24, No. 4, 1997, pp. 383-391.

[9] U. Kamps, "A Concept of Generalized Order Statistics," Elsevier Journal of Statistical Planning and Inference, Vol. 48, No. 1, 1995, pp. 1-23.

[10] U. Kamps, "Subranges of Generalized Order Statistics from Exponential Distributions," Fasciculi Mathematici, Vol. 28, 1998, pp. 63-70.

[11] M. Ragab, "Generalized Exponential Distribution: Moments of Order Statistics," Journal of Theoretical and Applied Statistics, Vol. 38, No. 1, 2004, pp. 29-41.

[12] R. D. Gupta and D. Kundu, "Generalized Exponential Distributions," Australian and New Zealand Journal of Statistics, Vol. 41, No. 2, 1999, pp. 173-188. doi:10.1111/1467-842X.00072

[13] G. Qiu and J. Wang, "Some Comparison between Generalized Order Statistics," Applied Mathematics - A Journal of Chinese Universities Series B, Vol. 22, No. 3, 2007, pp. 325-333. 
doi:10.1007/s11766-007-0310-6

[14] M. Garg, "On Generalized Order Statistics from Kumaraswamy Distribution," Tamsui Oxford Journal of Mathematical Sciences, Vol. 25, No. 2, 2009, pp. 153-166.
[15] P. Samuel, "Characterization of Distributions by Conditional Expectation of Generalized Order Statistics," Statistical Papers, Vol. 49, No. 1, 2008, pp. 101-108. doi:10.1007/s00362-006-0364-1 